\title{
Cor triatriatum dexter: antemortem diagnosis in an adult by cross sectional echocardiography
}

\author{
Anatole Trakhtenbroit, Pirzada Majid, Roxann Rokey
}

\begin{abstract}
Cor triatriatum dexter is a rare cardiac abnormality in which the right atrium is subdivided into two distinct chambers. This anomaly is generally attributed to the persistence of the right sinus venosus valve and it is frequently associated with severe malformations of other right heart structures. The antemortem diagnosis of the membrane may be difficult and its presence is often only established at necropsy. In a woman of 56 with Ebstein's anomaly the echocardiographic features of cor triatriatum dexter were examined before and during cardiac catheterisation. These investigations showed the position of the membrane, that there was no gradient between the two atrial chambers, and that there were perforations in the centre of the membrane.
\end{abstract}

Cor triatriatum dexter or septation of the right atrium to form a triatrial heart is a rare cardiac condition caused by persistence of the embryonic right valve of the sinus venosus. ${ }^{12}$ It is commonly associated with important abnormalities of other right heart structures. Only those with less severe involvement of the right heart survive childhood. ${ }^{2-5}$ Usually the abnormal membrane is first discovered at necropsy, but recent reports suggest the diagnosis can be established antemortem by cross sectional echocardiography. ${ }^{67}$ We describe a middle aged woman with echocardiographic findings of cor triatriatum dexter and Ebstein's anomaly. The anatomical location of the membrane and its physiological effects were established during a combined echocardiographic and cardiac catheterisation procedure and these results were confirmed at surgery.

\section{Case report}

A 56 year old woman was admitted for assessment of frank cyanosis and clubbing of all digits; these had been progressing slowly for 15 years. She sought medical attention when dyspnoea on exertion and fatigue increased in the two weeks before admission. She did not have palpitation, orthopnoea, chest pain, or oedematous feet.

The jugular venous pressure was elevated and a prominent $\mathrm{CV}$ wave of tricuspid regurgitation was noted. A right ventricular impulse was absent. The first heart sound was widely split and soft. The second heart sound was narrowed but not paradoxically split and the pulmonary component was soft. A right ventricular third heart sound was audible as was a II/VI early decrescendo systolic murmur over the lower left sternal border which increased with inspiration. The liver was enlarged and pulsatile.

The chest radiograph showed clear lung fields and a small aortic root. The atria and right ventricle were enlarged. An electrocardiogram showed normal sinus rhythm with a normal PR interval ( $200 \mathrm{~ms})$, a right ventricular conduction defect without QRS prolongation, and $a \mathrm{Q}$ wave in $\mathrm{V} 1$ and an $\mathrm{S}$ wave in $\mathrm{V} 5$ and $\mathrm{V} 6$. The left atrium was enlarged (fig 1).

CROSS SECTIONAL AND DOPPLER

ECHOCARDIOGRAMS

The situs of the atria and viscera was solitus with concordant atrioventricular and ventriculoarterial connections. A membrane measuring $7 \mathrm{~cm}$ in length divided the right atrium diagonally into two chambers forming a superomedial and inferolateral compartment. The membrane was very mobile during the cardiac cycle. During ventricular systole the central portion of the structure inverted towards the roof of the right atrium and during diastole it everted towards the right ventricle (fig 2). The tricuspid valve leaflets were displaced downward into the right ventricular

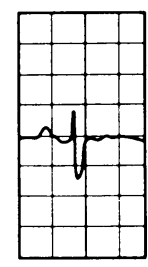
I

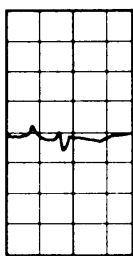

II

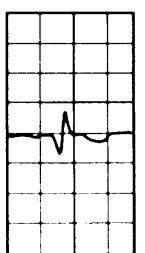

III
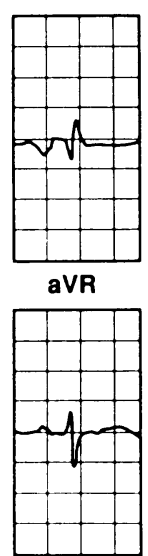

aVL

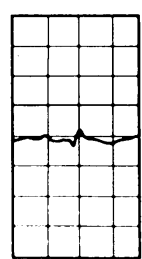

aVF

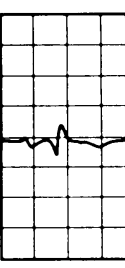

V1

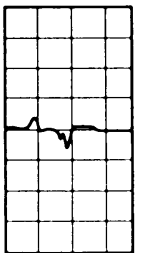

V2

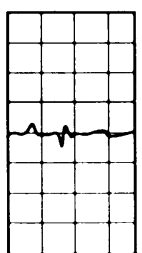

V3

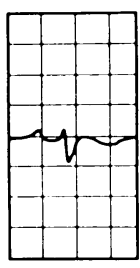

V4

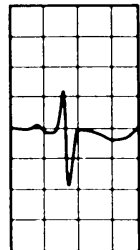

V5

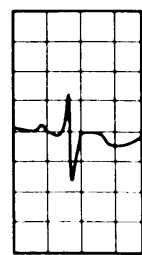

v6
Figure 1 Twelve lead electrocardiogram. 

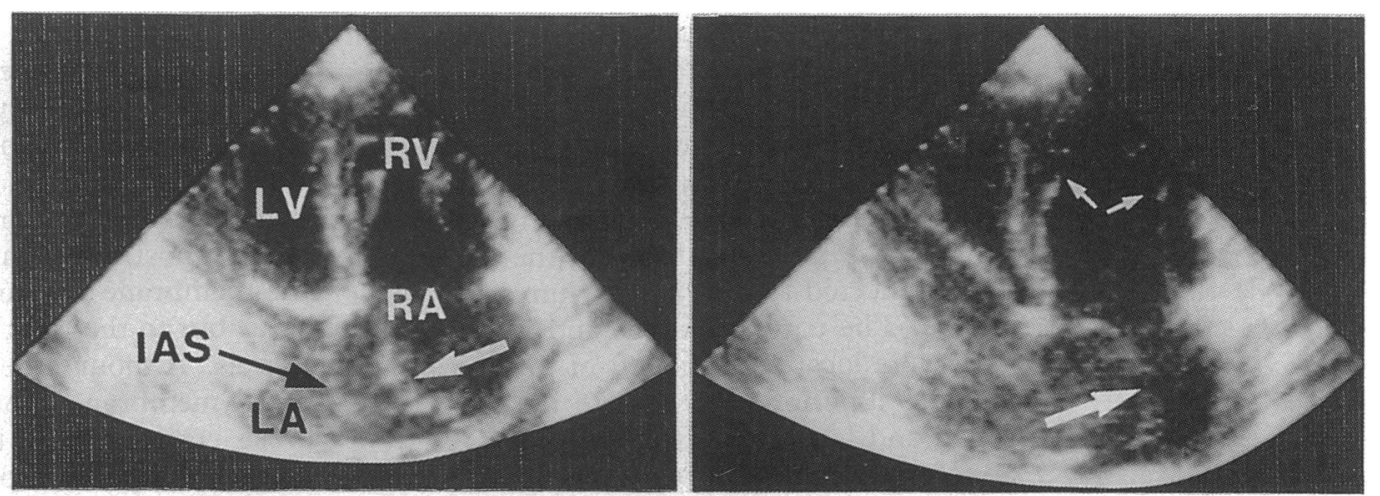

Figure 2 Apical four chamber echocardiogram of the heart during systole (left) and diastole (right). There was a large mobile membrane dividing the right atrium into two chambers (large arrow). The membrane inverted toward the roof of the right atrium during systole and everted toward the right ventricle during diastole. Displacement of the tricuspid valve consistent with Ebstein's anomaly was also apparent (small arrows). IAS, interatrial septum; $L A$, left atrium; $L V$, left ventricle; $R A$, right atrium; $R V$, right ventricle.

cavity. No significant obstruction of flow between the two right atrial chambers was detected on the Doppler echocardiographic examination.

On the basis of the physical examination and echocardiographic findings, Ebstein's anomaly and cor triatriatum dexter with no significant obstruction to venous flow was diagnosed.

\section{CARDIAC CATHETERISATION AND}

ANGIOCARDIOGRAPHY

A combined cardiac catheterisation and echocardiographic procedure was performed. The right femoral artery, right femoral vein, and right internal jugular vein were cannulated. The venous catheters were advanced into the superior and inferior venae cavae. Echocardiography of agitated saline injected into the inferior vena cava showed opacification of the superomedial right atrial compartment and shunting of contrast across the mid-interatrial septum into the left atrium. The contrast also streamed anterogradely into the lateral compartment through multiple areas in the central portion of the right atrial membrane. When contrast was injected in the superior vena cava, the inferolateral right atrial compartment opacified before the superomedial compartment. Retrograde filling through the central portion of the membrane resulted in complete opacification of the medial chamber within two cardiac cycles (fig 3 ).

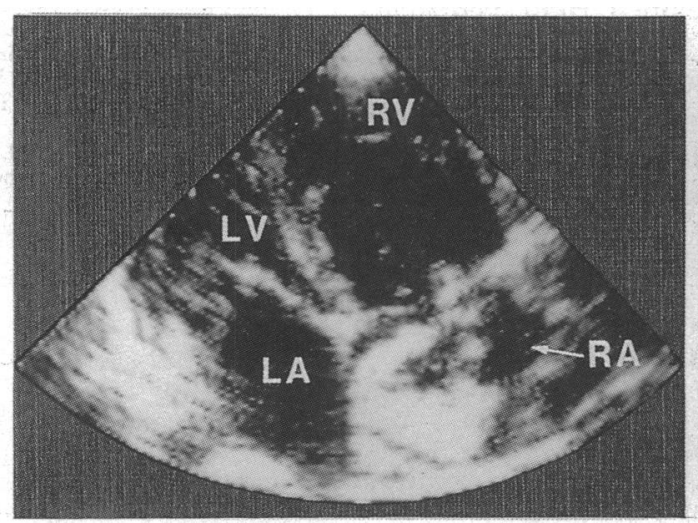

The superior vena cava catheter entered the right ventricle through the lateral right atrial compartment. The inferior vena cava catheter was seen in the medial right atrial chamber and easily passed through the mid-interatrial septum into the left atrium. Despite repeated attempts, neither catheter could be passed across the right atrial membrane to enter the opposite atrial compartment. Though the catheters could not be placed through the membrane, as predicted by the earlier Doppler examination, no significant gradient between the two compartments was detected. The right ventricular, pulmonary artery, and left atrial pressures were normal. A bidirectional shunt at the mid-atrial level of $3.25 \mathrm{l} / \mathrm{min}$ (right to left) and $0.251 / \mathrm{min}$ (left to right) was calculated from oximetric measurements and oxygen consumption.

Angiocardiography of the right heart showed the displaced tricuspid valve leaflets, enlarged right atrium and right ventricle, and tricuspid regurgitation. Despite multiple projections, the membrane seen by echocardiography could not be shown during angiography. There were no atherosclerotic lesions of the coronary arteries.

\section{FURTHER COURSE}

Surgical intervention for tricuspid valve repair or replacement, closure of the atrial septal defect, and resection of the membrane was

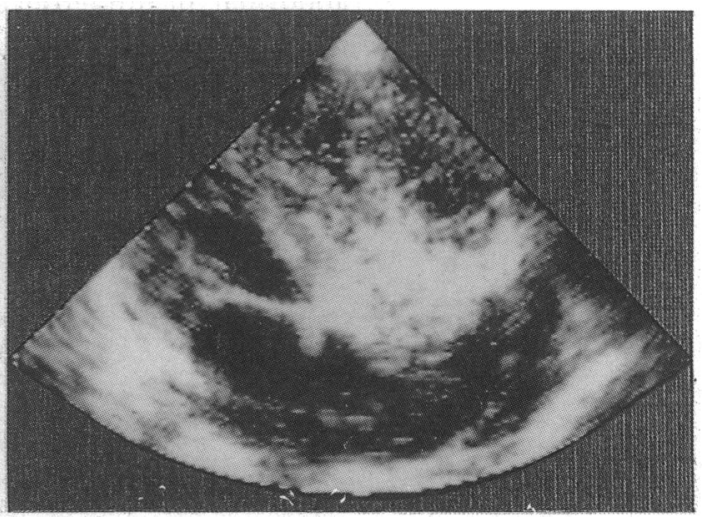

Figure 3 Modified apical four chamber echocardiograms obtained during cardiac catheterisation after injection of contrast through the inferior vena cava (left) and the superior vena cava (right). Injection of contrast through the inferior vena cava showed the initial opacification of the superomedial right atrial chamber and streaming of contrast through the central portion of the membrane into the inferolateral chamber. Injection of contrast through the superior vena cava showed opacification of the inferolateral right atrial chamber. $L A$, left atrium; $L V$, left ventricle; $R A$, right vena cava showed opacification; $R V$, right ventricle. 
recommended. At surgery, a large redundant fibrous membrane with multiple small centrally located fenestrations was found dividing the right atrium into two compartments. The membrane was attached inferiorly from the cristae terminalis to the inferior limbus of the interatrial septum. Anteriorly, the membrane was connected to the right atrium in a similar course. The coronary sinus and inferior vena cava were located to the left of the membrane and the superior vena cava to the right. The septal and lateral tricuspid valve leaflets were adherent to the right ventricular wall. There was an atrial septal defect of the patent foramen ovale type. Primary closure of the atrial septal defect, resection of the right atrial membrane, and insertion of a prosthetic tricuspid valve was performed. After operation the patient's cyanosis resolved and tricuspid regurgitation was no longer present.

\section{Discussion}

The membrane seen in the reported cases of cor triatriatum dexter is generally attributed to a persistence of the right sinus venosus valve. ${ }^{128}$ This valve forms during atrial development when the sinus venosus and other structure are progressively incorporated into the developing atria. By 12 weeks' development, the superior portion of the valve is absorbed into the cristae terminalis. The remainder of the right valve typically disappears. However, small remnants of the mid and inferior aspects of the valve occasionally persist, forming the eustachian valve of the inferior vena cava or the thebesian valve of the coronary sinus respectively. Failure of the membrane to regress normally, with subdivision of the right atrium into two distinct chambers, and no separately identifiable eustachian valve is called cor triatriatum dexter.

Necropsy data indicate a high incidence of congenital abnormalities, particularly of the right heart, in patients with cor triatriatum dexter..$^{2-5}$ These findings are attributed to the substantial redirection of blood by the right venous valve away from the right ventricle and into the left atrium during embryogenesis. ${ }^{257}$ The most common malformations include an abnormal or underdeveloped right ventricle and pulmonary artery stenosis or atresia. Abnormalities of the tricuspid valve also occur, including Ebstein's anomaly. ${ }^{9} 10$

To our knowledge the attachment of the membrane left of the superior vena cava but right of the inferior vena cava and coronary sinus seen in our patient has not been described before. In a review by Doucette and Knoblich the most common location of the membrane was to the right of the superior vena cava, coronary sinus, and inferior vena cava and the second most common pattern found was when the membrane was to the left of the coronary sinus but to the right of the other two venous vessels. ${ }^{2}$ Although the mid and inferior portion of the membrane in our patient most certainly arose entirely from the right sinus venosus valve, the superior aspect may have arisen from the persistence of a skewed septum secundum and the right sinus venosus valve. ${ }^{2}$

Typical findings of cor triatriatum dexter at cross sectional echocardiography were confirmed at necropsy in a neonate. ${ }^{7}$ In that patient, there were also two right atrial compartments divided by an extensive membrane with superoinferior attachment. Contrast injection through the inferior vena cava showed complete right to left shunting of blood across a patent foramen ovale. In our patient, echocardiography was also valuable in detecting the membrane and in further defining the presence of Ebstein's anomaly. Using this imaging technique during the catheterisation, we were able to determine the position of the membrane in relation to the great vessels whereas angiography did not show its presence. The echocardiographic procedure before and during catheterisation showed that there was no gradient between the two chambers on the Doppler examination and that there were perforations in the centre of the membrane on the contrast study.

1 Yater WM. Variations and anomalies of the venous valves of the right atrium of the human heart. Arch Pathol the right atrium

2 Doucette J, Knoblich R. Persistent right valve of the sinus venosus. Arch Pathol 1963;75:105-12.

3 Folger GM. Supravalvular tricuspid stenosis. Am J Cardiol 1968;21:81-7.

4 Jones RN, Niles NR. Spinnaker formation of sinus venosus valve. Circulation 1968;38:468-73.

5 Hansing CE, Young WP, Rowe GG. Cor triatriatum dexter: persistent right sinus venosus valve. Am J Cardiol 1972 30:559-64.

6 Battle-Diaz J, Stanley P, Kratz C, Fouron JC, Guerin R, Davignon A. Echocardiographic manifestations of persistence of the right sinus venosus valve. Am J Cardiol 1979; 43:850-3.

7 Alboliras ET, Edwards WD, Driscoll DJ, Seward JB. Cor triatriatum dexter: two-dimensional echocardiographic diagnosis. J Am Coll Cardiol 1987;9:334-7.

8 Moore KL. The developing human: clinically oriented embryology. Philadelphia: WB Saunders, 1974:248-9.

9 Verel D, Pilcher J, Hynes DM. Cor triatrium dexter. $B$ Heart J 1970;32:714-6.

10 Gerlis LM, Anderson RH. Cor triatriatum dexter with imperforate Ebstein's anomaly. Br Heart J 1976;38: 108-11. 